\title{
Multiple Imputation of missing values in exploratory factor analysis of multidimensional scales: estimating latent trait scores
}

\author{
Urbano Lorenzo-Seva ${ }^{1 *}$ y Joost R. Van Ginkel ${ }^{2}$ \\ 1 CRAMC (Research Center for Behavior Assessment); Department of Psychology; Universitat Rovira i Virgili (Tarragona, Spain) \\ 2 Leiden University (Leiden, The Netherlands)
}

\begin{abstract}
Título: Imputación múltiple de valores perdidos en el análisis factorial exploratorio de escalas multidimensionales: estimación de las puntuaciones de rasgos latentes.

Resumen: Los investigadores con frecuencia se enfrentan a la difícil tarea de analizar las escalas en las que algunos de los participantes no han respondido a todos los ítems. En este artículo nos centramos en el análisis factorial exploratorio de escalas multidimensionales (es decir, escalas que constan de varias de subescalas), donde cada subescala se compone de una serie de ítems de tipo Likert, y el objetivo del análisis es estimar las puntuaciones de los participantes en los rasgos latentes correspondientes. En este contexto, se propone un nuevo enfoque para hacer frente a las respuestas faltantes que se basa en (1) la imputación múltiple de las respuestas faltantes y (2) la rotación simultánea de las muestras de datos imputados. Se ha aplicado el método en una muestra de datos reales en que las respuestas que faltantes fueron introducidas artificialmente siguiendo un patrón real de respuestas faltantes, y un estudio de simulación basado en conjuntos de datos artificiales. Los resultados muestran que nuestro enfoque (en concreto, Hot-Deck de imputación múltiple seguido de rotación Consensus Promin) es capaz de calcular correctamente la puntuación factorial estimada incluso para los participantes que tienen valores perdidos.

Palabras clave: Valores perdidos; Imputación Hot-Deck; Imputación Predictive mean matching; Imputación múltiple; Consensus Rotation; Puntuaciones factoriales; Análisis factorial exploratorio.
\end{abstract}

Abstract: Researchers frequently have to analyze scales in which some participants have failed to respond to some items. In this paper we focus on the exploratory factor analysis of multidimensional scales (i.e., scales that consist of a number of subscales) where each subscale is made up of a number of Likert-type items, and the aim of the analysis is to estimate participants' scores on the corresponding latent traits. We propose a new approach to deal with missing responses in such a situation that is based on (1) multiple imputation of non-responses and (2) simultaneous rotation of the imputed datasets. We applied the approach in a real dataset where missing responses were artificially introduced following a real pattern of non-responses, and a simulation study based on artificial datasets. The results show that our approach (specifically, Hot-Deck multiple imputation followed of Consensus Promin rotation) was able to successfully compute factor score estimates even for participants that have missing data.

Key words: Missing data; Hot-Deck imputation; Predictive mean matching imputation; Multiple imputation; Consensus Rotation; Factor scores; Exploratory factor analysis.

\section{Introduction}

The ultimate aim of psychological testing is to estimate the score of a person in one or more latent psychological variables (known as latent traits). The estimate is based on a person's answers to a set of items (i.e. a psychological test): each item in the test helps the person to report a particular facet of his/her own personality or how (s)he would react or feel in a particular situation. Frequently, these items are Likerttype items: responses to items are based on a binary or a graded format. With this aim (i.e., to estimate factor scores from responses to Likert-type items), psychological test data obtained in a large sample is typically analyzed using exploratory factor analysis (EFA). However, as responses to Likerttype items cannot be regarded as continuous-unbounded variables, typical linear factor analysis is inappropriate in this situation. An alternative to linear factor analysis is the nonlinear Underlying Variable Approach (UVA; see, for example, Mislevy, 1986; Moustaki, Joreskog, \& Mavridis, 2004).

The UVA uses a two-level approach: on the first level, it is assumed that the observed item response arises as a result of a categorization of an underlying response variable; on the

* Dirección para correspondencia [Correspondence address]:

Urbano Lorenzo-Seva, Departament de Psicologia; Universitat Rovira i

Virgili; Carretera de Valls s/n; 43007, Tarragona (Spain).

E-mail: urbano.lorenzo@urv.cat second level, it is assumed that the linear model holds for these underlying responses. Parameters are estimated from the bivariate tetrachoric/polychoric tables between pairs of item scores. The simplest and most usual approach is known as the heuristic solution (Bock \& Aitkin, 1981): item thresholds are estimated from the marginals of the table, and the tetrachoric/polychoric correlations are estimated from the joint frequency cells. Then, the usual factor analysis of the polychoric correlation matrix provides estimates of item loadings and residual variances. Once the estimates have been obtained, they can be reparameterized so that the model is reported in the most usual (multidimensional) Item Response Theory (IRT) form (see, for example, Ferrando \& LorenzoSeva, 2013). Finally, factor scores on the latent variables can be estimated. One popular approach is to compute expected a posteriori (EAP) estimators, which have good properties that other estimators do not usually have (Muraki \& Engelhard, 1985). It must be noted that, in order to compute these factor-score estimates for a particular individual in the sample, (s)he must have provided an answer to each item in the psychological test. However, a typical difficulty when analyzing the responses of a sample of participants is missing data: some respondents fail to respond to some items (item nonresponse).

A particular person may refuse to answer an item because of interaction between the characteristics of the person and the characteristics of the item. For example, a person 
with low lexical abilities may not respond to an item that includes a complex word. Rubin (1976) formalized the three mechanisms that underlie the missing data process: (a) missing completely at random (MCAR), (b) missing at random (MAR), and (c) missing not at random (MNAR). The MCAR values occur when the probability that a particular value is missing in the data set is independent of all other (observed and non-observed) variables. As a consequence, the missing values occur randomly for all variables in the data set. The MAR values occur when the probability that a value is missing depends on the observed variables in the data set, but not on the unobserved variables. The MNAR values occur when the probability that a value is missing depends on unobserved variables.

Even if the problem of item nonresponse is as old as psychological testing, it can still be an obstacle in studies nowadays. For example, in a recent study on marital happiness, Johnson and Young (2011) observed that the percentage of missing responses was highest for questions about sexual behavior (23\%) and total household income (19\% to $27 \%$ ). Schlomer, Bauman and Card (2010) recently studied how researchers currently cope with missing responses in applied research (Vol. 55 of the Journal of Counseling Psychology, 2008), and concluded that, despite the prevalence of missing data and the existence of recommendations for taking these data into account, this journal had not yet done so. Nowadays, research journals are publishing papers on best practices and recommendations about missing responses (see, for example, Cuesta, Fonseca, Vallejo, \& Muñiz,, 2013, Graham, 2009, Kleinke, Stemmler, Reinecke, \& Lösel, , 2011).

In order to cope with missing responses, incomplete response patterns are often deleted from the sample (listwise deletion or pairwise deletion). Even though this is easy to do, deleting cases with missing responses could lead to bias in the parameter estimates of the factor model (for example, loading values). In addition, because some responses are missing, the estimated score on the latent variable cannot be computed for those individuals with incomplete response patterns. One of the techniques recommended for handling item nonresponse is imputation: the missing values are filled in so that a complete data set is created and then analyzed with traditional methods of analysis. However, single imputation methods are considered outdated (see for example, Schafer \& Graham, 2002). While single imputation can lead to approximately unbiased point estimates, estimated standard errors are systematically underestimated (Rässler, Rubin, \& Zell, 2013).

A more elaborate approach to filling in missing responses is the multiple imputation (MI) method (Rubin, 1978): instead of creating a single complete data set, a number of copies are created by imputation. Then, each copy of data is analyzed independently, and the final outcome is obtained as a combination of the outcomes obtained in the copies of data. One advantage of $\mathrm{MI}$ is that the final standard errors of these parameter estimates are based on both (a) the standard errors of the analysis of each data set and (b) the dispersion of pa- rameter estimates across data sets. As MI accounts for the random fluctuations between each imputation, it provides accurate standard errors and therefore accurate inferential conclusions. If MI is a general method, it can be applied using different techniques (i.e., the complete copies of data can be generated using different approaches). Nowadays, the use of MI is quite popular in applied research in psychology: a Google search for the terms psychology "multiple imputation" produces about 131,000 hits.

Within the framework of IRT, missing values are frequently treated as if they were the result of an incomplete testing design (i.e., subsets of items administered to different respondents) (see, for example, DeMars, 2003). The resulting incomplete data can be analyzed with IRT models and estimates of latent abilities. However, as Huisman and Molenaar (2001) point out, this strategy for handling item-nonresponse cannot be used in every situation. When this approach is not feasible, imputation of missing data appears as an advisable alternative. Imputation of missing data in IRT has been studied in the context of unidimensional models (Ayala, Plake, \& Impara, 2001; DeMars, 2003; Finch, 2008, 2011; Huisman \& Molenaar, 2001; Sijtsma \& Van der Ark, 2003). Recently, Wolkowitz and Skorupski (2013) proposed a single imputation approach intended to estimate statistical properties of items but not factor scores. Finally, no research has yet been undertaken in the framework of multidimensional IRT.

MI has already been proposed in the context of confirmatory factor analysis, and can be computed using, for example, Mplus (Muthén \& Muthén, 1998-2011). In this context, the copies of data created using MI are analyzed independently but with one restriction: they share the same hypothesis for the factor solution in the population. The fact that all the copies of data share the same hypothesis means that the outcomes of the copies of data are comparable, and may consequently be combined to produce one final outcome. However, Mplus does not allow MI to be computed in the the context of EFA: as there is no hypothesis of the factor solution in the population (because of the exploratory nature of the analysis), the outcomes obtained in different copies of data are not necessarily comparable. This means that the EFA outcomes that are produced for each data copy cannot be directly combined to produce one final outcome. This last difficulty seems to indicate that MI cannot be used in EFA.

We start by presenting a new approach based on the MI of missing responses in psychological tests in the context of EFA. Our approach focuses on the exploratory nonlinear factor analysis (i.e., the underlying variable approach) of Likert-type items in multidimensional tests. The main aim of our method is to make it possible to compute estimates of factor scores for all individuals in the sample. In addition, our method does not assume any particular missing response mechanism. Finally, we assess the effectiveness of the procedure in two simulation studies: (1) a simulation study based on a real dataset; and (2) a simulation study in which different characteristics of datasets were manipulated. 


\section{Procedure to obtain estimates of latent trait scores for ordinal data when data is missing}

The procedure that we propose is based on five main steps that are explained in detail below and summarized in Figure
1. None of the analyses included in the five steps is new and they can be found in the literature. The merit of our proposal is to point out how they can be used to compute multidimensional exploratory factor analysis when some responses are missing.

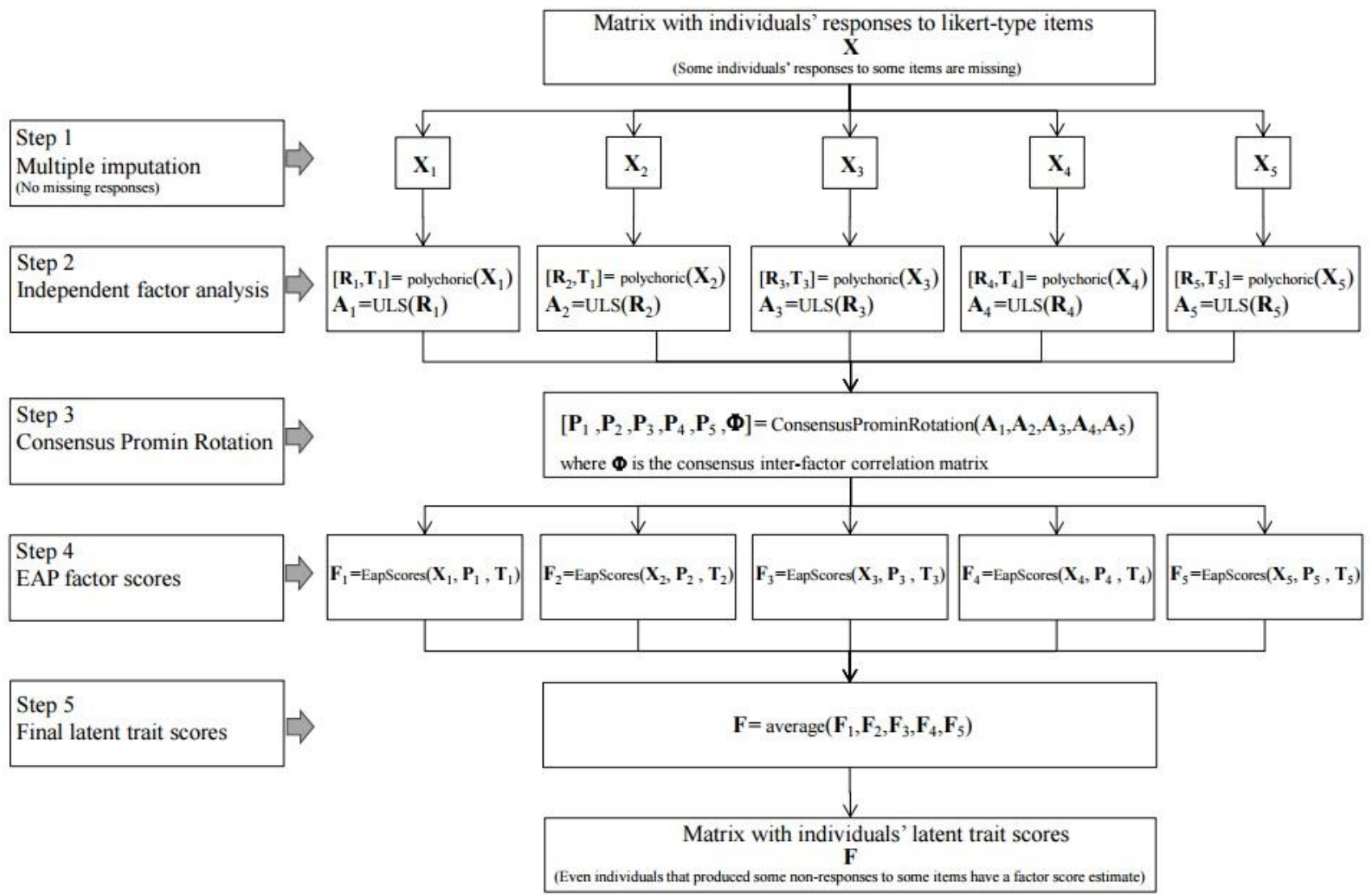

Figure 1. Procedure to obtain estimates of latent trait scores for ordinal data when data is missing from the dataset.

\section{Step 1: Multiple imputation}

The problem that needs to be solved is how to fill in the missing values of a participant in a multidimensional psychological test (i.e., scales that consist of a number of subscales) in which each subscale is made up of a number of Likerttype items. For this purpose, various MI approaches can be used. In our simulation studies presented below, we use two approaches: Hot-deck Multiple Imputation (HD-MI), and Predictive mean matching (PMM-MI).

Single hot-deck imputation was developed for item nonresponse in the Income Supplement of the Current Population Survey, initiated in 1947 (Ono \& Miller, 1969). A recent review of different techniques of hot-deck imputation can be found in Andridge and Little (2010). Hot-deck replaces missing values in incomplete cases (donees) with observed values from donors in the same data set to create a complete data set. In some versions, the donor is selected randomly from a set of potential donors (the donor pool). In other versions, a single donor is identified and values are imputed from that individual, who is usually the "nearest neighbor" based on some metric. Siddique and Belin (2007) point out the following benefits of hot-deck imputation: (1) imputations tend to be realistic since they are based on values observed elsewhere; (2) imputations will not be outside the range of possible values; and (3) it is not necessary to define an explicit model for the distribution of the missing values. They conclude that, because of the simplicity of the hot-deck approach and these desirable properties, it is a popular method of imputation, especially in large-sample survey settings where there is a large pool of donors. As psychological tests are frequently multidimensional scales (i.e., scales that consist of a number of subscales) that consist of a number of Likerttype items, hot-deck imputation is a simple and convenient procedure for dealing with missing responses: (a) hot-deck imputation can easily be implemented even if the number of 
items is large (a typical situation in multidimensional scales); (b) a large sample is available from which potential donors can be taken; and (c) all the imputations will be in the range of specific values used in the Likert-type items.

Single hot-deck imputation can be generalized to become a multiple imputation procedure: Hot-deck Multiple Imputation (HD-MI), also known as K-nearest-neighbors hot-deck imputation. This is an imputation technique in which missing values in incomplete cases (donees) are replaced with observed values from donors in the same data set to create $K$ complete data sets. HD-MI has been shown to improve the simplest approaches, and it has remained a popular option in many applications (Aittokallio, 2010). Like single HD imputation, HD-MI is a simple and convenient procedure for dealing with missing responses.

Predictive mean matching (PMM) (Rubin, 1986) could at some extend be defined as a hot-deck imputation method: the main difference is that observed values for $Y$ are regressed on a set of observed variables $X$. Then, predicted values for $Y$ are calculated for all $Y$ using the regression parameters calculated for the observed data. Finally, missing $Y$ values are imputed using observed values of $Y$ whose predicted values most closely match the predicted values of the respondents with missing data. The set of predictor variables $X$ to be used to predict variable $Y$ must be correlated with variable $Y$. Each variable $Y$ to be imputed can use a different set of predictor variables $X$.

When we applied HD-MI, we selected the $K$ nearest neighbors (i.e., the donors) to the donee. The selection was made taking into consideration all the individuals of the sample that produced responses for the same set of items as the donee: the $K$ participants with the lowest Euclidean distance are taken as the donors. Once the $K$ donors for each donee have been selected, $K$ copies of the original data are generated in which donees' missing responses are replaced with the corresponding donors' responses. PMM-MI uses the procedure explained above to create $K$ copies of data as well. In this way, $K$ complete versions of the data set are obtained. In our simulation study presented below, we used $K$ $=5$ with acceptable results.

As our approach is not related to a particular MI method, researchers can use the MI procedures that we tested in our simulation studies or others that are available in the literature. MI can be computed by software packages such as SPSS, $\mathrm{R}$ (see Amelia II package available at http://gking.harvard.edu/amelia), or Matlab (for example, function knnimpute available in Bioinformatics Toolbox).

\section{Step 2: Independent exploratory factor analysis}

Once the data have been multiply imputed, each copy is independently analyzed using EFA. As already explained, the nonlinear UVA is appropriate for analyzing the data. In the most usual approach (see e.g. Mislevy, 1986), the item thresholds are estimated from the marginals in the table and the tetrachoric/polychoric correlations are estimated from the joint frequency cells. So, routine factor analysis of the tetrachoric/polychoric correlation matrix provides the estimates of the loading values. In this way, for each copy of data, we obtain (a) the item thresholds, (b) the polychoric correlation matrix, and (c) the matrix of loading values. It must be noted that the decision on how many $r$ factors to extract (one factor for each latent trait) has to be the same for the $K$ copies of data. The factors can be extracted using Unweighted Least Squares (ULS), for example. After this step, $K$ unrotated loading matrices $\mathbf{A}_{k}$ are obtained.

Researchers can use the $R$ package polycor to compute polychoric correlation matrices (http://cran.rproject.org/web/packages/polycor/). In addition, the $R$ package psych makes it possible to compute different factor loading estimates

(http://cran.r-project.org/web/packages/psych/).

\section{Step 3: Consensus factor rotation}

In EFA of a single dataset, the loading matrix is typically rotated to maximize factor simplicity (Kasier, 1974) using an orthogonal or an oblique rotation method. However, in this step of the analysis, $K$ loading matrices $\mathbf{A}_{k}$ need to be rotated. In our situation, the independent rotation that maximizes simplicity in each loading matrix $\mathbf{A}_{k}$ has an important drawback: the freedom of the final position of rotated factors means that the rotated factor solutions may turn out to be non-comparable between the $K$ copies of data. A (semi) confirmatory factor analysis would not have this drawback: the hypothetical loading matrix that is proposed in the population model is used as a kind of target to the $K$ factor solutions. However, in an EFA such a common hypothesis (or target) does not exist. To avoid the drawback, the $K$ factor loading matrices $\mathbf{A}_{k}$ have to be simultaneously orthogonally (or obliquely) rotated so that they are both (a) factorially simple, and (b) as similar to one another as possible. For the orthogonal rotation, Consensus Varimax can be computed (see, for example, Kiers, 1997). For the oblique rotation, Consensus Promin (Lorenzo-Seva, Kiers, \& ten Berge, 2002) is available. Both consensus rotations are based on a previous Generalized Procrustes Rotation (GPR). Let $\mathbf{A}_{k}$ be the set $(k=1 \ldots K)$ of unrotated loading matrices of order $m \times r$ obtained by factor analysis with $m$ variables and $r$ factors retained. This set of loading matrices is orthogonally rotated by GPR (ten Berge, 1977) by minimizing,

$$
g\left(\mathbf{S}_{1}, \ldots, \mathbf{S}_{p}\right)=\sum_{k=1}^{K} \sum_{l=1}^{K}\left\|\mathbf{A}_{k} \mathbf{S}_{k}-\mathbf{A}_{l} \mathbf{S}_{l}\right\|^{2}
$$


over $\mathbf{S}_{1}, \ldots, \mathbf{S}_{k}$, subject to $\mathbf{S}_{k} \mathbf{S}_{k}=\mathbf{S}_{k} \mathbf{S}_{k}=\mathbf{I}$. So the set of loading matrices $\mathbf{A}_{k} \mathbf{S}_{k}$ shows optimal agreement in the least squares sense. The Consensus Promin rotation consists of applying Promin (Lorenzo-Seva, 1999) to the mean of the matched loading matrices $\mathbf{A}_{k} \mathbf{S}_{k}$, thus minimizing

$$
\boldsymbol{f}_{K}(\mathbf{U})=\operatorname{promin}\left(\frac{1}{K}\left(\sum_{k=1}^{K} \mathbf{A}_{k} \mathbf{S}_{k}\right) \mathbf{U}\right)
$$

with $\mathbf{U}$ subject to $\operatorname{diag}\left(\mathbf{U}^{-1} \mathbf{U}^{-1 \prime}\right)=\mathbf{I}$. The oblique loading matrices $\mathbf{P}_{k}$ of order $m \times r$ are computed as,

$$
\mathbf{P}_{k}=\mathbf{A}_{k} \mathbf{S}_{k} \mathbf{U}
$$

As far as we know, neither Consensus Varimax nor Consensus Promin rotations have been specifically programmed in $R$ language. However, researchers can use the various $R$ packages available to obtain a consensus rotation. For example, GPR is available in the $\operatorname{proc} G P A$ package (http://www.inside-

r.org/packages/cran/shapes/docs/procGPA ), and Promin rotation is available in the $P C o v R$ package (http://www.inside-.org/packages/cran/PCovR/docs/pro-

min; Vervloet et al., 2015). After the consensus rotation, pattern matrices of the $K$ copies of data are comparable, and can be used to compute estimates of latent trait scores.

\section{Step 4: Estimates of latent trait scores}

Because the items in the psychological test are frequently Likert-type items, an appropriate procedure should be used to estimate the $r$ latent trait scores. One popular approach is to compute expected a posteriori (EAP) estimators (Muraki \& Engelhard, 1985). In the context of our multiple imputation method, EAP estimates of the $r$ latent trait scores must be computed for the $K$ copies of the data. For each copy of the data, the corresponding item scores (with donees' missing responses replaced with the corresponding donors' responses), item thresholds, and the rotated loading matrix are used to compute the $r$ EAP scores. For each individual, $K$ EAP estimates are computed related to the $r$ latent traits.

Although EAP factor scores are not frequently used in applied research, they can be computed using an $R$ package: Latent Trait Models under IRT (ltm) (http://www.insider.org/packages/cran/ltm/docs/factor.scores).

\section{Step 5: Final latent trait scores}

Once the $K$ estimates of the $r$ latent trait scores are available for each individual, the average of the $K$ estimates of each individual is computed so that the final estimates of the $r$ latent trait scores can be obtained.

\section{Simulation study based on a real dataset}

In this section, we present an illustrative example of how the multiple imputation method followed by simultaneous rotation performs with a dataset in which missing data are artificially introduced. The aim is to assess whether the imputation method can obtain reasonably good estimators of the latent trait scores for incomplete data. The study has four main steps: (a) first, for a particular psychological test, it detects the pattern of missing values obtained in a real situation; (b) second, it introduces missing data into a dataset that was initially complete; (c) then, it computes the estimates of the latent trait scores using the original dataset (i.e., the dataset that is free of missing data), and the estimates of the latent trait scores after introducing artificial missingness; (d) and, finally, it compares the estimators obtained in both situations to assess the performance of the imputation method proposed. In addition to the multiple imputation method, we included a simplistic alternative imputation method that is frequently used in real research. In the section below we describe the simulation study in detail.

\section{Obtaining missing-data patterns from incomplete data}

To study the pattern of missing values in a real situation, a sample of 747 individuals ( $51 \%$ women) were administered the Overall Personality Assessment Scale (OPERAS) (VigilColet et al., 2013). OPERAS is a short measure for the fivefactor model personality traits: Extraversion (EX), Emotional Stability (ES), Conscientiousness (CO), Agreeableness $(\mathrm{AG})$, and Openness to Experience (OE). Each personality trait is measured with 7 items, and the participant must indicate the level of agreement with a sentence by using a fivepoint scale that goes from "fully disagree" (1) to "fully agree" (5). The test was administered in the traditional paperand-pencil format. A sentence at the end of the test reminded the participants to review the test so that they would spot missing data. Two participants had more than 10 missing values: as they left more than $25 \%$ of items unanswered, these two participants were eliminated from the sample.

Even though the respondents were reminded to review their responses, on 65 occasions (out of 26,145) an item was not answered. All scales had missing data (with frequencies ranging from 10 to 17 ), and the maximum number of missing values was observed in EX. A total number of 55 individuals had incomplete response patterns $(7.4 \%$ of the sample): 2 participants had 3 missing values, 6 participants had 2 missing values, and 47 participants had only 1 missing value. These outcomes were taken as the pattern of missing data to be observed in OPERAS in a real situation. This pattern is used in the next step to introduce artificial missing data into a complete data set. 
OPERAS was administered to a second sample of 745 participants (34\% women). However, this second sample answered an on-line format of the test. In this version of the test a single item was presented on a computer screen at a time, and the computer refused to continue with the next item until a response had been given. With the on-line version, it was impossible to skip questions (i.e., non-responses could not be produced by the responder). Please note that OPERAS was actually developed by its authors in both paper-and-pencil and on-line formats.

The aim was to artificially introduce missing data into this second sample using the missing-data patterns from the first sample. Specifically, we aimed to introduce the missing values in the same kind of participants as the set of participants who had given non-responses in the first sample, and in exactly the same items. The first step was to select the 55 participants in the second sample that were most similar to the 55 participants with missing data in the first: we computed the Euclidean distance of the responses of the first participant who produced non-response in the first sample with respect to the responses of the 745 participants in the second sample, and selected the participant in the second sample who was most similar to that participant in the first sample. Please note that the Euclidean distance was computed using only the items to which the participant in the first sample actually produced a response. The second step was to artificially introduce in the participant selected from the second sample the same non-responses as the participant from the first sample (i.e., we deleted the responses in exactly the same items in which a non-response was observed). The procedure was replicated then for the second participant who produced a non-response in the first sample, in order to select the most similar participant from the second sample (now of 744 participants), and the non-responses observed in the participant of the first sample were also introduced in the participant of the second sample. This two-step procedure was replicated until we had 55 participants in the second sample with exactly the same non-responses as the 55 participants in the first sample.

At this point we had (1) a sample of 745 participants that had not produced any non-responses, and (2) the same sample in which it was suspected that 55 participants would have produced a non-response if the computer had allowed them to and who had had 65 non-responses artificially introduced (following the pattern of non-responses observed in the sample that was administered the paper-and-pencil format test). In the rest of the document, we shall refer to the first sample as the Full Response (FR) sample, and the second as the Artificial Non-Response (ANR) sample.
Computing the estimates of latent trait scores in the FR sample is a typical analysis that presents no difficulties. However, computing estimates of latent trait scores in the ANR sample is impossible, unless a specific method is used to deal with non-responses. In the section below we compute EAP estimates in the FR sample and use multiple imputation to compute EAP estimates in the ANR sample. We also use a popular single imputation procedure to assess whether our multiple imputation improves the performance of this single imputation method.

\section{Computing the estimates of the latent trait scores}

In order to compute the estimates of the latent trait scores in the FR sample, we used the program FACTOR (Lorenzo-Seva \& Ferrando, 2013). We computed the polychoric correlation matrix. The value of the KMO index was .87 , which indicated that the correlation matrix was suitable for factor analysis. Optimal parallel analysis (Timmerman \& Lorenzo-Seva, 2011) suggested that five factors could be extracted. We extracted the five factors using unweighted least squares extraction, and obtained a CFI index of .98. To maximize factor simplicity, we computed Promin rotation (Lorenzo-Seva, 1999). The salient loading values of items in the rotated pattern were in accordance with the scales EX, ES, $\mathrm{CO}, \mathrm{AG}$, and OE. Finally, we computed the estimates of the five latent trait scores using the EAP estimator. The means and variances of the estimates are shown in Table 1 , in the columns labeled True. The table shows the statistics for the whole sample, for the subsample of the 55 participants with artificial missing data, and the subsample of 690 participants with complete response patterns. The outcomes of the whole sample show that, as expected with the EAP estimator, means are close to zero, and variances are lower than 1 . The same pattern is observed for the subsample of 690 participants whose responses are unchanged. However, the means of the subsample of the 55 participants with artificial missing data can help us to understand the kind of participants that were expected not to respond to all items in this test. These participants generally had low scores on OE, EX, and AG. This probably means that they did not understand some of the items (low score on OE), were shy to ask for help (low score on $\mathrm{EX}$ ), or did not care enough about the instructions to review their response patterns (low score on AG). Except for the scores on EX, this subsample was quite homogeneous in this pattern (low variances). As the pattern of missing values observed in the data seems to be dependent on the observed variables included in the model, the data-missing mechanism for this data set seems to be MNAR. 
Table 1. Mean and variances (printed in parentheses) for the true and the estimate of factor scores in the five personality factors.

\begin{tabular}{|c|c|c|c|c|c|c|c|c|c|c|c|c|}
\hline \multirow[t]{3}{*}{ Factor } & \multicolumn{4}{|c|}{ Factor scores for the whole sample } & \multicolumn{4}{|c|}{$\begin{array}{l}\text { Factor scores for the subsample of } \\
\text { individuals with missing data } \\
\qquad N=55\end{array}$} & \multicolumn{4}{|c|}{$\begin{array}{l}\text { Factor scores for the subsample of individuals with- } \\
\text { out missing data } \\
\qquad N=690\end{array}$} \\
\hline & \multirow[t]{2}{*}{ True } & $\begin{array}{l}\text { Estim } \\
\text { imput: }\end{array}$ & $\begin{array}{l}\text { ates base } \\
\text { ation met }\end{array}$ & & \multirow[t]{2}{*}{ True } & \multicolumn{3}{|c|}{$\begin{array}{l}\text { Estimates based on } \\
\text { imputation methods }\end{array}$} & \multirow[t]{2}{*}{ True } & \multicolumn{3}{|c|}{ Estimates based on imputation methods } \\
\hline & & PMM-MI & HD-MI & Mode-I & & PMM-MI & HD-MI & Mode-I & & PMM-MI & HD-MI & Mode-I \\
\hline EX & $\begin{array}{c}0.012 \\
(0.929)\end{array}$ & $\begin{array}{c}0.009 \\
(0.915)\end{array}$ & $\begin{array}{c}0.011 \\
(0.928)\end{array}$ & $\begin{array}{c}0.011 \\
(0.928)\end{array}$ & $\begin{array}{l}-0.154 \\
(1.036)\end{array}$ & $\begin{array}{l}-0.180 \\
(1.049)\end{array}$ & $\begin{array}{l}-0.159 \\
(1.006)\end{array}$ & $\begin{array}{l}-0.160 \\
(0.971)\end{array}$ & $\begin{array}{c}0.025 \\
(0.919)\end{array}$ & $\begin{array}{c}0.024 \\
(0.903)\end{array}$ & $\begin{array}{c}0.024 \\
(0.921)\end{array}$ & $\begin{array}{c}0.024 \\
(0.924)\end{array}$ \\
\hline ES & $\begin{array}{c}0.019 \\
(0.955)\end{array}$ & $\begin{array}{c}0.021 \\
(0.970)\end{array}$ & $\begin{array}{c}0.019 \\
(0.955)\end{array}$ & $\begin{array}{c}0.019 \\
(0.955)\end{array}$ & $\begin{array}{l}-0.004 \\
(0.637)\end{array}$ & $\begin{array}{l}-0.043 \\
(0.646)\end{array}$ & $\begin{array}{l}-0.016 \\
(0.636)\end{array}$ & $\begin{array}{l}-0.005 \\
(0.633)\end{array}$ & $\begin{array}{c}0.021 \\
(0.981)\end{array}$ & $\begin{array}{c}0.026 \\
(0.997)\end{array}$ & $\begin{array}{c}0.021 \\
(0.981)\end{array}$ & $\begin{array}{c}0.021 \\
(0.982)\end{array}$ \\
\hline $\mathrm{CO}$ & $\begin{array}{c}0.022 \\
(0.899)\end{array}$ & $\begin{array}{c}0.022 \\
(0.900)\end{array}$ & $\begin{array}{c}0.022 \\
(0.898)\end{array}$ & $\begin{array}{c}0.022 \\
(0.899)\end{array}$ & $\begin{array}{l}-0.032 \\
(0.692)\end{array}$ & $\begin{array}{l}-0.044 \\
(0.661)\end{array}$ & $\begin{array}{l}-0.029 \\
(0.654)\end{array}$ & $\begin{array}{l}-0.001 \\
(0.644)\end{array}$ & $\begin{array}{c}0.026 \\
(0.917)\end{array}$ & $\begin{array}{c}0.027 \\
(0.920)\end{array}$ & $\begin{array}{c}0.026 \\
(0.919)\end{array}$ & $\begin{array}{c}0.024 \\
(0.920)\end{array}$ \\
\hline AG & $\begin{array}{c}0.027 \\
(0.760)\end{array}$ & $\begin{array}{c}0.028 \\
(0.753)\end{array}$ & $\begin{array}{c}0.027 \\
(0.759)\end{array}$ & $\begin{array}{c}0.027 \\
(0.759)\end{array}$ & $\begin{array}{l}-0.140 \\
(0.481)\end{array}$ & $\begin{array}{l}-0.128 \\
(0.439)\end{array}$ & $\begin{array}{l}-0.134 \\
(0.467)\end{array}$ & $\begin{array}{l}-0.119 \\
(0.472)\end{array}$ & $\begin{array}{c}0.041 \\
(0.780)\end{array}$ & $\begin{array}{c}0.041 \\
(0.776)\end{array}$ & $\begin{array}{c}0.040 \\
(0.781)\end{array}$ & $\begin{array}{c}0.039 \\
(0.781)\end{array}$ \\
\hline OP & $\begin{array}{c}0.015 \\
(0.811)\end{array}$ & $\begin{array}{c}0.016 \\
(0.810)\end{array}$ & $\begin{array}{c}0.015 \\
(0.806)\end{array}$ & $\begin{array}{c}0.015 \\
(0.802)\end{array}$ & $\begin{array}{l}-0.439 \\
(0.775)\end{array}$ & $\begin{array}{l}-0.460 \\
(0.770)\end{array}$ & $\begin{array}{l}-0.417 \\
(0.686)\end{array}$ & $\begin{array}{l}-0.379 \\
(0.699)\end{array}$ & $\begin{array}{c}0.051 \\
(0.797)\end{array}$ & $\begin{array}{c}0.053 \\
(0.795)\end{array}$ & $\begin{array}{c}0.050 \\
(0.800)\end{array}$ & $\begin{array}{c}0.046 \\
(0.798)\end{array}$ \\
\hline
\end{tabular}

In order to compute the estimates of the latent trait scores in the NR sample, we used three imputation methods to handle the missing data. The methods we used were:

1. Hot-Deck Multiple Imputation (HD-MI) (see above). We used five copies of data. When subjecting the copies of the data to factor analysis, we used the same methods as the ones used when the FR sample was analysed. The only difference was that instead of Promin rotation (useful when a single dataset is analyzed), we computed Consensus Promin rotation (useful when simultaneously rotating a number of datasets).

2. Predictive Mean Matching Multiple Imputation (PMMMI) (see above). Again, we used five copies of the data, and we used the same procedure as with HD-MI to factor analyze the $K$ copies of data obtained.

3. Single imputation of the mode of the item (Mode-I). Any missing value in the dataset was replaced with the mode of the item where the non-response was observed. We used the mode (instead of the mean or the median) because we aimed to supply one of the answers that was already on the response scale of the item (i.e., the values 1 , $2,3,4$, and 5). After the imputation of modes, we replicated the methods used when the FR sample was subject to factor analysis.

The means and variances of the estimates are shown in Table 1, in the columns labeled PMM-MI, HD-MI and ModeI. As factor score estimates are computed from the information obtained after the rotation of the factor loading matrix, a possible criticism of imputation is that it affects the estimates related to the whole sample (not only the subsample of participants in which non-responses are observed), and consequently it might change the estimates of participants who do not have missing responses. To determine whether this criticism can be applied to our data analysis, Table 1 shows the statistics for (1) the whole sample, (2) the subsample of individuals who have artificial missing data, and (3) the subsample of individuals who do not have missing data. As can be observed in the table, the three imputation methods closely replicated the same values (in terms of mean and variance) when analyzing the FR sample (i.e., when there are no missing data at all) in (a) the whole sample, and (b) the subsample of individuals who did not have missing data. However, the estimates for the subsample of participants who had artificial missing data were generally replicated best when a multiple imputation method was used. As can be expected, the worst imputation approach was Mode-I, whereas HD-MI and PMM-MI performed quite similarly. Table 2 shows the correlations between (a) the factor score estimates obtained in the FR sample (i.e., when there were no missing data), and (b) the factor score estimates obtained in the NR sample (i.e., when artificial missing data were introduced into the dataset). In terms of correlation, HD-MI performed slightly better than the others.

We also computed the bias defined as the difference between (1) the factor score estimates obtained in the NR sample after (multiple) imputation, and (2) the factor score estimates obtained in the FR sample. In addition, we computed the Root Mean Square of Residuals (RMSR) between both estimates: the observed values were .079. .029, and .041, respectively, for PPM-MI, HD-MI, and Mode-I. The mean bias (and its corresponding 95\% confidence interval), the variance of the bias, and the RMSR are shown in Table 3. The outcomes in the table are presented for both the subsample of participants with artificial missing data, and the subsample of participants without missing data. When the subsample of participants with missing data was considered, the lowest bias was observed for HD-MI (not significantly different from 
zero), whereas the more homogenous bias was observed for PMM-MI (in terms of variance and RMSR). When the subsample of participants without missing data was considered, the three imputation methods produced very accurate esti- mates. However, PMM-IM was the approach that showed the largest RMSR: in this regard, PMM-IM seems to be the method that most affected the factor score estimates of the participants without missing data.

Table 2. Correlations between the true scores and the estimates based on different imputation methods.

\begin{tabular}{|c|c|c|c|c|c|c|c|c|c|}
\hline \multirow[t]{2}{*}{ Factor } & \multicolumn{3}{|c|}{ Total sample } & \multicolumn{3}{|c|}{$\begin{array}{l}\text { Subsample of individuals } \\
\text { with missing data } \\
N=55\end{array}$} & \multicolumn{3}{|c|}{$\begin{array}{l}\text { Subsample of individuals } \\
\text { without missing data } \\
N=690\end{array}$} \\
\hline & PMM-MI & HD-MI & Mode-I & PMM-MI & HD-MI & Mode-I & PMM-MI & HD-MI & Mode-I \\
\hline EX & .9969 & .9997 & .9996 & .9922 & .9967 & .9958 & .9974 & 1.0000 & 1.0000 \\
\hline ES & .9917 & 9998 & 9998 & .9803 & .9968 & .9967 & .9923 & 1.0000 & 1.0000 \\
\hline $\mathrm{CO}$ & .9996 & .9996 & 9991 & .9935 & .9931 & .9851 & 9999 & 1.0000 & 1.0000 \\
\hline $\mathrm{AG}$ & .9952 & .9996 & 9995 & .9760 & .9907 & .9904 & .9961 & 1.0000 & 1.0000 \\
\hline OP & .9990 & .9987 & .9969 & .9932 & .9827 & .9602 & .9994 & 1.0000 & .9998 \\
\hline
\end{tabular}

Table 3. Descriptive statistics of estimation bias (estimate score minus true score) for different imputation methods.

\begin{tabular}{|c|c|c|c|c|c|c|}
\hline \multirow[t]{2}{*}{ Statistics } & \multicolumn{3}{|c|}{$\begin{array}{c}\text { Subsample of individuals } \\
\text { with incomplete response patterns } \\
N=55\end{array}$} & \multicolumn{3}{|c|}{$\begin{array}{c}\text { Subsample of individuals } \\
\text { with complete response patterns } \\
\qquad N=690\end{array}$} \\
\hline & PMM-MI & HD-MI & Mode-I & PMM-MI & HD-MI & Mode-I \\
\hline Mean & -0.056 & 0.024 & 0.111 & 0.0012 & -0.0006 & -0.0006 \\
\hline $95 \%$ CI & $(-0.104 ;-0.007)$ & $(-0.028 ; 0.077)$ & $(0.043 ; 0.180)$ & $(-0.001 ; 0.004)$ & $(-0.001 ; 0.000)$ & $(-0.001 ; 0.000)$ \\
\hline Variance & 0.037 & 0.043 & 0.074 & 0.0057 & 0.0002 & 0.0002 \\
\hline RMSR & 0.200 & 0.208 & 0.292 & 0.076 & 0.013 & 0.016 \\
\hline
\end{tabular}

\section{Simulation study based on artificial datasets}

On the basis of the theoretical considerations and results from research discussed in the sections above, we hypothesize that our multiple imputation approach will outperform the single imputation approach when used to estimate true factor scores. To study the comparative performance of two multiple imputation procedures (HD-MI and PMM-MI) and estimate the true factor score of individuals under different circumstances, we performed a simulation study based on artificial data.

\section{Data construction}

The simulated data were generated with a linear common factor model, where the resulting continuous variables were categorized to yield ordered polytomous observed variables. The linear common factor model included both major and minor factors, as may well be the case with real-world data, on the basis of the middle model by Tucker, Koopman and Linn (1969). This approach was adopted in earlier research on the common factor model (see for example, Timmerman \& Lorenzo-Seva, 2011). In the simulation study, the population correlation matrix of the continuous variables $\mathbf{R}^{*}$ pop was taken as

$\mathbf{R}_{p o p}^{*}=w_{m a} \boldsymbol{\Lambda}_{m a} \boldsymbol{\Phi}_{m a} \boldsymbol{\Lambda}_{m a}{ }^{\prime}+w_{m i} \boldsymbol{\Lambda}_{m i} \boldsymbol{\Lambda}_{m i}{ }^{\prime}+w_{m n} \mathbf{I}_{J}$,

where $\Lambda_{m a}\left(J \times Q_{m a}\right)$ and $\Lambda_{m i}\left(J \times Q_{m i}\right)$ are major and minor loading matrices, respectively, with $Q_{m a}$ and $Q_{m i}$ being the number of major and minor factors, and $J$ the number of observed variables; $\boldsymbol{\Phi}_{m a}\left(Q_{m a} \times Q_{m a}\right)$ is the inter-factor correla- tion between major factors; $\mathbf{I}_{J}(J \times J)$ is the identity matrix, reflecting the covariance matrix of the unique parts of the variables; $w_{m a}, w_{m i}$ and $w_{m n}$ are weights that make it possible to manipulate $\sigma_{m a}^{2}, \sigma_{m i}^{2}$ and $\sigma_{u n}^{2}$, the variances of the major, minor and unique parts of the correlation matrix, respectively. In our study, these variances were kept constant so that $\sigma_{m a}^{2}=.64$, and $\sigma_{m i}^{2}=.10$. In addition, the number of major and minor factors was also kept constant: we considered two major factors and six minor factors. The interfactor correlation between major factors was systematically .30. Each simulated continuous data matrix $\mathbf{X}^{*}(N \times J)$, with sample size $N$, was obtained by randomly drawing $N$ vectors from a multivariate normal distribution $\mathrm{N}\left(\mathbf{0}, \mathbf{R}^{*}{ }_{p o p}\right)$. Subsequently, each element $x_{n j}$ of the polytomous simulated data matrix $\mathbf{X}(N \times J)$ was obtained from the element $x_{n j}^{*}$ of the continuous data matrix $\mathbf{X}^{*}$ using prespecified thresholds $\tau_{c}\left(\tau_{c}\right.$ $=\tau_{0}, \ldots, \tau_{C}$, with $C=5$ the number of response categories), with $x_{n j}=c$ if $\tau_{c-1}<x_{n j}^{*} \leq \tau_{c}$. In real situations the item responses are non-symmetrically distributed so the distribution of the variables was manipulated to be systematically skewed in our datasets. For each single factor, half of the variables were skewed in the opposite direction to mimic differences in item difficulty in real scales and the thresholds were chosen such that the expected proportion of observations in categories $c=1, \ldots, C$ were $[0.05,0.60,0.20,0.10,0.05]$.

The various conditions in the experimental design were manipulated so that they represented conditions present in 
empirical research. The sample size was varied $(N=500$, 1,000 and 2,000) and the number of observed variables per major factor was also varied $(M=5$ and 10). This means that, as the number of major factors was kept constant to 2 , the number of observed variables in the model was $J=10$ and 20.

For each $\mathbf{X}$, we computed the estimated latent trait scores as follows: (a) we computed the corresponding polychoric correlation matrix $\mathbf{R}$; (b) we extracted two factors using unweighted least squares factor analysis, and (c) we computed estimated latent trait scores using the EAP estimator for each individual in $\mathbf{X}$. These estimated latent trait scores were considered the true estimated latent trait scores $\left(\boldsymbol{\theta}_{t}\right)$ that would be obtained if the data contained no missing values.

\section{Simulation of artificial missing data}

Once data matrix $\mathbf{X}$ was available, we introduced different amounts of artificial missing data in order to obtain $\mathbf{Y}$ (i.e., the same dataset as $\mathbf{X}$, but with missing data). The proportion of missing data was manipulated to be $G=.05, .10$, and .15. The three mechanisms that underlie the missing data process (MCAR, MNAR, and MAR) were simulated in order to produce data with artificial missing data. To generate MCAR data, for each $x_{i j}$ value in $\mathbf{X}$, a uniform number between 0 and $1(U)$ was randomly drawn. If the value of $U$ was less than or equal to $G$, the item response $y_{i j}$ was deleted. To generate MNAR data, we computed the total scale score $(S)$ of each individual as the addition of the observed responses of each participant in $\mathbf{X}$. Then we computed $P($ missing $\mid S)=G(1-\varphi(S))$, where $\varphi(S)$ is the additive inverse of the normal cumulative density function. Once $P($ missing $\mid S)$ had been calculated, a uniform number between 0 and $1(U)$ was randomly drawn. If the value of $U$ was less than or equal to $P(\operatorname{missing} \mid S)$, the item response $y_{i j}$ was deleted. To generate MAR data, we computed a normally distributed variable $V$ that was correlated .5 with $\boldsymbol{\theta}_{t}$. Then we computed $P$ (missing $\mid \zeta)=G(1-\varphi(V))$, where $\varphi(V)$ is the additive inverse of the normal cumulative density function. Once $P$ (missing $\mid V$ ) had been calculated, a uniform number between 0 and $1(U)$ was randomly drawn. If the value of $U$ was less than or equal to $P(\operatorname{missing} \mid V)$, the item response $y_{i j}$ was deleted.

It must be noted that from each matrix $\mathbf{X}$ (i.e., a matrix of individuals' responses without missing data), 9 different matrices $\mathbf{Y}$ (i.e., a matrix of individuals' responses with missing data) were computed: 3 different values of $G \times 3$ missing data mechanisms.

\section{Imputation of missing data}

Once matrices $\mathbf{Y}$ were available, we proceeded to apply the same imputation methods that we had used in the previous simulation study: Hot-Deck Multiple Imputation (HD-MI), Predictive Mean Matching Multiple Imputation (PMM-MI), and Single imputation of the mode of the item (Mode-I). For each $\mathbf{Y}$, we computed the estimated latent trait scores as follows: (a) we computed the corresponding polychoric correlation matrix; (b) we extracted two factors using unweighted least squares factor analysis, and (c) we computed estimated latent trait scores using the EAP estimator for each individual in each $\mathbf{Y}$. These estimated latent trait scores were considered the estimated latent trait scores $(\boldsymbol{\theta})$ that could be obtained when the data contain missing values.

\section{Dependent variable}

We computed 500 replicates of the study. This resulted in 2 (number of observed variables per major factor) $\times 3$ (percentage of missing responses) $\times 3$ (mechanism to produce missing responses) $\times 500$ (replicates) $=27,000$ simulated data sets with artificially introduced missing responses. As the size of the datasets with missing responses was $N=500,1000$, or 2,000 , the number of participants simulated in the study was $31,500,000$. For each participant, the estimated latent trait scores were computed for both factors in each data set (i.e., a total of $64,000,000$ estimated latent trait scores were computed), where the missing values were imputed using the three approaches discussed above: HD-MI, PMM-MI and Mode-I. To assess the performance of each imputation approach, we computed the bias of the estimated latent trait scores: true estimated latent trait scores minus estimated latent trait scores $\left(\boldsymbol{\theta}_{t}-\boldsymbol{\theta}\right)$. To assess the accuracy, we computed the average bias. To assess the efficiency, we computed the standard deviation of bias.

\section{Results and conclusion of the simulation study}

Table 4 shows the mean and standard deviation of bias of the three imputation approaches. Overall, it can be seen that Mode-I was the imputation approach with the largest average bias (with estimated factor scores lower than the true ones), and the largest standard deviation (i.e., less efficiency). While both multiple imputation approaches performed quite similarly, HD-MI offered the best accuracy and efficiency. 
Table 4. Average of bias obtained in the simulation study based on artificial data. Standard deviations of bias are printed in parenthesis.

\begin{tabular}{|c|c|c|c|c|c|c|c|c|c|c|}
\hline \multirow{2}{*}{$\begin{array}{l}\text { Condition } \\
\text { Overall }\end{array}$} & & \multirow[b]{2}{*}{0} & \multicolumn{2}{|c|}{ Mode-I } & \multicolumn{3}{|c|}{ HD-MI } & \multirow[b]{2}{*}{0} & \multicolumn{2}{|c|}{ PMM-MI } \\
\hline & & & .003037 & $(.1825)$ & 0 & .000126 & $(.1477)$ & & -.000580 & $(.1548)$ \\
\hline \multirow[t]{3}{*}{ Missing-response mechanism } & MCAR & 0 & .001922 & $(.1764)$ & 0 & .000121 & $(.1422)$ & 0 & -.000429 & $(.1492)$ \\
\hline & MAR & 0 & .004584 & $(.1904)$ & 0 & .000376 & $(.1565)$ & 0 & -.000583 & $(.1626)$ \\
\hline & MNAR & 0 & .002604 & $(.1805)$ & 0 & -.000120 & $(.1439)$ & 0 & -.000728 & $(.1522)$ \\
\hline \multirow[t]{3}{*}{ Sample Size } & 500 & 0 & .002724 & $(.1820)$ & 0 & .000194 & $(.1507)$ & 0 & -.000493 & $(.1547)$ \\
\hline & 1000 & 0 & .003102 & $(.1827)$ & 0 & .000040 & $(.1474)$ & 0 & -.000635 & $(.1539)$ \\
\hline & 2000 & 0 & .003082 & $(.1826)$ & 0 & .000152 & $(.1470)$ & 0 & -.000574 & $(.1552)$ \\
\hline \multirow[t]{2}{*}{ Number of items per factor $(m / r)$} & 5 & 0 & .001710 & $(.1963)$ & 0 & -.000051 & $(.1562)$ & 0 & -.000670 & $(.1697)$ \\
\hline & 10 & 0 & .004364 & $(.1677)$ & 0 & .000302 & $(.1386)$ & 0 & -.000491 & $(.1382)$ \\
\hline \multirow[t]{3}{*}{ Percentage of missing responses } & 5 & 0 & .001827 & $(.1248)$ & 0 & -.000122 & $(.0960)$ & 0 & -.000521 & $(.1031)$ \\
\hline & 10 & 0 & .003195 & $(.1811)$ & 0 & -.000044 & $(.1440)$ & 0 & -.000705 & $(.1512)$ \\
\hline & 15 & 0 & .004089 & $(.2271)$ & 0 & .000543 & $(.1884)$ & 0 & -.000514 & $(.1959)$ \\
\hline
\end{tabular}

The most difficult situations for HD-MI were when (a) the missing data mechanism was MAR, (b) the number of observed variables per factor was large, and (c) the percentage of missing data was large. PMM-MI showed a consistent tendency to overestimate the true values. The most difficult situations of PMM-MI were when (a) the missing data mechanism was MNAR, (b) the number of observed variables per factor was low, and (c) the percentage of missing responses was large.

Overall, these results show that single imputation of missing values should not be used (i.e., Mode-I in our simulation study), and that multiple imputation based on the HotDeck approach (i.e., HD-MI in our simulation study) was the most accurate and efficient approach. In order to get further insight into the interaction among the conditions used in the simulation study, we further studied the results obtained with the most successful approach (i.e., HD-MI). Table 5 shows the average bias in the various conditions related to HD-MI. When missing responses were due to MAR, the bias was largest when the number of variables per factor was large and the percentage of missing responses was large; this bias was even greater when the sample size was low. When missing responses were due to MCAR, the bias was largest when the sample size was low, the number of items per factor large, and the percentage of missing responses large. When missing responses were due to MNAR, the bias was lower than when missing responses were due to MAR or MCAR. However, bias increased when the percentage of missing responses was large, the number of items per factor was large, and the sample size was large.

Table 5. Average of bias obtained related to HD-MI approach depicted for manipulated conditions of the simulation study.

\begin{tabular}{|c|c|c|c|c|c|}
\hline Sample size & Number of items per factor & Percentage of missing responses & MCAR & MAR & MNAR \\
\hline \multirow[t]{6}{*}{500} & 5 & 5 & .00001 & -.00019 & -.00006 \\
\hline & & 10 & -.00002 & -.00039 & -.00004 \\
\hline & & 15 & .00010 & -.00074 & -.00025 \\
\hline & 10 & 5 & -.00010 & -.00040 & -.00021 \\
\hline & & 10 & .00014 & .00046 & -.00018 \\
\hline & & 15 & .00115 & .00314 & .00104 \\
\hline \multirow[t]{6}{*}{1000} & 5 & 5 & .00014 & -.00035 & -.00025 \\
\hline & & 10 & -.00032 & -.00050 & -.00039 \\
\hline & & 15 & -.00006 & -.00085 & .00042 \\
\hline & 10 & 5 & .00076 & -.00030 & .00039 \\
\hline & & 10 & .00029 & .00075 & -.00151 \\
\hline & & 15 & .00059 & .00302 & -.00111 \\
\hline \multirow[t]{6}{*}{2000} & 5 & 5 & .00014 & -.00004 & .00015 \\
\hline & & 10 & .00018 & -.00023 & .00035 \\
\hline & & 15 & .00032 & .00008 & -.00026 \\
\hline & 10 & 5 & -.00039 & -.00035 & -.00074 \\
\hline & & 10 & .00064 & .00034 & -.00090 \\
\hline & & 15 & -.00065 & .00280 & .00129 \\
\hline
\end{tabular}




\section{Discussion}

In EFA, researchers usually have to deal with missing data: for some reason, some participants leave some items unanswered. While the performance of multiple imputation with continuous data has been extensively studied, much less work has been done on the performance of multiple imputation with ordinal data (Finch, 2008). We suggest how the multiple imputation approach can be used in the context of EFA. It should be pointed out that while multiple imputation has been studied in unidimensional situations (see, for example, Finch, 2008, 2011), our approach is useful in multidimensional situations: to our knowledge no previous work has been done on this specific situation, which is frequent in real applied research. The key step in our procedure is to simultaneously rotate the $K$ copies of data obtained after multiple imputation, so that the $K$ factor scores for each individual are comparable (i.e., the average between the $K$ factor score estimates of an individual can be computed to obtain the final factor score estimation of the individual).

We carried out simulations based on real data and artificial datasets. In the study with real data, we used two samples: one sample in which a personality test was administered in the traditional pencil and paper format, and which had missing data; and a sample in which the same personality test was administered with computer software that did not allow for non-responses. The results in this simulation study suggested that our approach was actually successful, and produced better factor score estimates than single imputation methods.

In the study with the artificial dataset, we manipulated three missing data mechanisms (MCAR, MAR, and MNAR), the sample size, the proportion of items per factor, and the percentage of missing responses. The results of this study suggest that single imputation (Mode-I in our study) is not an advisable option, and that HD-MI is the most accurate and efficient of the approaches used. HD-MI was less accurate when MAR was the mechanism responsible of the nonresponses, than when MCAR or MNAR were the responsible mechanisms of the nonresponses. In the same way, PMMMI was less efficient when MAR was the mechanism responsible of the nonresponses. The conclusion is that, of the three missing data mechanisms, MAR seems to be the most difficult one to deal with.

In the simulation study, our approach was tested with two multiple imputation methods: Hot-deck (Ono \& Miller, 1969), and Predictive Mean Matching (Rubin, 1986). Overall, Hot-deck Multiple Imputation seemed to perform slightly better in our dataset than Predictive Mean Matching Multiple Imputation. In addition, it should be pointed out that single imputation of the mode the items was the approach that performed worst.

Our paper proposes a multiple imputation approach to deal with missing responses, with particular focus on the procedure for obtaining latent trait estimates. However, other approaches can be found in the literature. Yuan, Marshall and Bentler (2002) proposed a unified approach to exploratory factor analysis that included missing values and was based on generalizing the maximum likelihood approach under constraints in order to assess statistical properties of estimates of factor loadings and error variances. However, they did not specifically deal with the difficulty of computing factor scores. Yuan and $\mathrm{Lu}(2008)$ provided the theory and application of the 2-stage maximum likelihood procedure for structural equation modeling with missing data (see also Yuan \& Zhang, 2012, and Yuan \& Savalei, 2014). Their approach is especially advisable if the data mechanism is MAR. If the missing data mechanism is unknown, they advise that auxiliary variables be included in the analysis to make the missing data mechanisms more likely to be MAR. From our point of view, it cannot be easily assumed that the MAR mechanism plays a role in nonresponses to psychological tests. A psychological test is composed of a number of items: even if all the items in the same scale are expected to have a latent variable in common, each item is related to a specific facet of the latent variable. What is more, if two items are related to exactly the same facet of a latent variable, then one of the items is redundant and should not have been in the test from the very beginning. The response to a psychological item may be missing because of the content of the specific facet the item is measuring or some specific characteristic of the item itself (for example, the item includes a word that is ambiguous for some individuals). None of these characteristics depends on the other items in the scale. From this point of view, the factor model cannot be estimated only from the available data. For this reason, the MAR mechanism cannot easily be assumed as the nonresponse mechanism in the context of psychological tests. Our proposal does not assume any missing data mechanism: it is based on sharing the information given by the participants who responded to the item for which a response was missing. The only assumption in our approach is that individuals who had already produced similar responses would tend to produce responses that were similar to those of the unanswered items. This assumption is more acceptable in the context of psychological tests.

Wolkowitz and Skorupski (2013) proposed a method for imputing response options for missing data based on multiple-choice assessments but state that it is intended for test development planning purposes, and that additional research is needed before it can be used to operationally score a test.

On limitation of using multiple imputation approach (based on HD or PMM) when unique cases (i.e., cases that are dissimilar to all others in the data set) are present in the data set: in this situation there would be no donor available. As Myers (2011) points out, this situation is more likely to be observed in small samples. In addition, as our approach is addressed to analyze Liker-type items using UVA, a minimum of 200 observations seems advisable in order to obtain stable polychoric correlation estimates (see Chen \& Choi, 2009). As a conclusion, our approach should only be used when a large sample is available. 
There are several $R$ language packages available to researchers interested in computing our approach to missing values in the context of multidimensional EFA. We have programmed our approach in Matlab: we shall be glad to share our Matlab functions with interested researchers. Finally, we have implemented the multiple imputation methods studied in this paper in FACTOR 10.1 (Lorenzo-Seva \& Ferrando, 2013), a stand-alone program for Windows for fitting exploratory and semiconfirmatory factor analysis and IRT

\section{References}

Aittokallio, T. (2010). Dealing with missing values in large-scale studies: microarray data imputation and beyond. Briefings in bioinformatics, 11, 253264. doi:10.1093/bib/bbp059.

Andridge, R. R., \& Little, R. J. (2010). A review of Hot Deck imputation for survey non-response. International Statistical Review, 78, 40-64. doi:10.1111/j.1751-5823.2010.00103.x.

Ayala, R. J., Plake, B. S., \& Impara, J. C. (2001). The impact of omitted responses on the accuracy of ability estimation in item response theory. Journal of educational measurement, 38, 213-234. doi:10.1111/j.17453984.2001.tb01124.x.

Bock, R. D., \& Aitkin, M. (1981). Marginal maximum likelihood estimation of item parameters: an application of the EM algorithm. Psychometrika, 46, 443-459. doi:10.1007/BF02293801.

Chen, J., \& Choi, J. (2009). A comparison of maximum likelihood and expected a posteriori estimation for polychoric correlation using Monte Carlo simulation. Journal of Modern Applied Statistical Methods, 8(1), 32.

Cuesta, M., Fonseca, E., Vallejo, G., \& Muñiz, J. (2013). Datos perdidos y propiedades psicométricas en los test de personalidad. Anales de Psicología, 29(1), 285-292. doi:10.6018/analesps.29.1.137901.

DeMars, C. (2003, April). Missing data and IRT item parameter estimation. Paper presented at the annual meeting of the American Educational Research Association, Chicago, IL.

Ferrando, P.J. \& Lorenzo-Seva, U. (2013). Unrestricted item factor analysis and some relations with item response theory. Technical Report. Department of Psychology, Universitat Rovira $i$ Virgili, Tarragona. Retrieved from http://psico.fcep.urv.cat/utilitats/factor.

Finch, H. (2008). Estimation of item response theory parameters in the presence of missing data. Journal of Educational Measurement, 45, 225-245. doi:10.1111/j.1745-3984.2008.00062.x.

Finch, H. (2011). The Use of Multiple Imputation for Missing Data in Uniform DIF Analysis: Power and Type I Error Rates. Applied Measurement in Education, 24, 281-301. doi:10.1080/08957347.2011.607054.

Graham, J. W. (2009). Missing data analysis: Making it work in the real world. Annual review of psychology, 60, 549-576. doi: 10.1146/annurev.psych.58.110405.085530

Huisman, M., \& Molenaar, I. W. (2001). Imputation of missing scale data with item response models. In Essays on item response theory (pp. 221244). Springer New York. doi:10.1007/978-1-4613-0169-1_13.

Johnson, D. R., \& Young, R. (2011). Toward best practices in analyzing datasets with missing data: Comparisons and recommendations. Journal of Marriage and Family, 73, 926-945. doi:10.1111/j.1741-3737.2011.00861.x.

Kaiser, H. F. (1974). An index of factorial simplicity. Psychometrika, 39, 3136. doi:10.1007/BF02291575

Kiers, H. A. (1997). Techniques for rotating two or more loading matrices to optimal agreement and simple structure: A comparison and some technical details. Psychometrika, 62, 545-568. doi:10.1007/bf02294642.

Kleinke, K., Stemmler, M., Reinecke, J., \& Lösel, F. (2011). Efficient ways to impute incomplete panel data. AStA Advances in Statistical Analysis, 95, 351-373. doi:10.1007/s10182-011-0179-9.

Lorenzo-Seva, U. (1999). Promin: a method for oblique factor rotation. Multivariate Behavioral Research, 34, 347-365. doi:10.1207/S15327906MBR3403_3.

Lorenzo-Seva, U., \& Ferrando, P. J. (2013). FACTOR 9.2: A Comprehensive Program for Fitting Exploratory and Semiconfirmatory Factor
Models. The program, a demonstration, and a short manual are available at: http://psico.fcep.urv.cat/utilitats/factor

Acknowledgements.- The research was partially supported by a grant from the Catalan Ministry of Universities, Research and the Information Society (2014 SGR 73) and by a grant from the Spanish Ministry of Education and Science (PSI2014-52884-P).

Analysis and IRT Models. Applied Psychological Measurement, 37, 497-498. doi:10.1177/0146621613487794.

Lorenzo-Seva, U.; Kiers, H. A. L.; ten Berge, J. M. F. (2002). Techniques for oblique factor rotation of two or more loading matrices to a mixture of simple structure and optimal agreement. British Journal of Mathematical of Statistical Psychology, 55, 337-360. doi:10.1348/000711002760554624.

Mislevy, R. J. (1986). Recent developments in the factor analysis of categorical variables. Journal of educational statistics, 11, 3-31. doi:10.3102/10769986011001003.

Moustaki, I., Joreskog, K., \& Mavridis, D. (2004). Factor models for ordinal variables with covariate effects on the manifest and latent variables: a comparison of LISREL and IRT approaches. Structural equation modelling, 11, 487-513. doi:10.1207/s15328007sem1104_1.

Muraki, E., \& Engelhard, G. (1985). Full-information item factor analysis: Applications of EAP scores. Applied Psychological Measurement, 9, 417430. doi:10.1177/014662168500900411.

Muthén, L. K., \& Muthén, B. O. (1998-2011). Mplus User's Guide. (Sixth ed.). Los Angeles, CA: Muthén \& Muthén.

Myers, T. A. (2011). Goodbye, listwise deletion: Presenting hot deck imputation as an easy and effective tool for handling missing data. Communication Methods and Measures, 5(4), 297-310. doi:10.1080/19312458.2011.624490.

Ono, M., \& Miller, H. P. (1969). Income nonresponses in the current population survey. In Proceedings of the Social Statistics Section, American Statistical Association, 277-288.

Rässler, S., Rubin, D. B., \& Zell, E. R. (2013). Imputation. Wiley Interdisciplinary Reviews: Computational Statistics, 5, 20-29. doi:10.1002/wics.1240.

Rubin, D. B. (1976). Inference and missing data. Biometrika, 63, 581-592. doi:10.1093/biomet/63.3.581.

Rubin, D. B. (1978). Multiple imputations in sample surveys-a phenomenological Bayesian approach to nonresponse. In Proceedings of the Section on Survey Research Methods, American Statistical Association, 2034.

Rubin, D. B. (1986). Statistical matching using file concatenation with adjusted weights and multiple imputations. Journal of Business \& Economic Statistics, 4, 87-94. doi:10.1080/07350015.1986.10509497.

Schafer, J. L., \& Graham, J. W. (2002). Missing data: our view of the state of the art. Psychological methods, 7, 147. doi: 10.1037/1082-989x.7.2.147.

Schlomer, G. L., Bauman, S., \& Card, N. A. (2010). Best practices for missing data management in counseling psychology. Journal of Counseling Psychology, 57, 1-10. doi:10.1037/a0018082.

Siddique, J., \& Belin, T. R. (2007). Multiple imputation using an iterative hot-deck with distance-based donor selection. Statistics in medicine, 27, 83-102. doi:10.1002/sim.3001.

Sijtsma, K., \& Van der Ark, L. A. (2003). Investigation and treatment of missing item scores in test and questionnaire data. Multivariate Behavioral Research, 38, 505-528. doi:10.1207/s15327906mbr3804_4.

Ten Berge, J. M. (1977). Orthogonal Procrustes rotation for two or more matrices. Psychometrika, 42, 267-276. doi:10.1007/BF02294053.

Timmerman, M.E.; Lorenzo-Seva, U. (2011). Dimensionality assessment of ordered polytomous items with parallel analysis. Psychological Methods, 16, 209-220. doi:10.1037/a0023353. 
Tucker, L. R., Koopman, R. F., \& Linn, R. L. (1969). Evaluation of factor analytic research procedures by means of simulated correlation matrices. Psychometrika, 34, 421-459. doi:10.1007/BF02290601.

Vervloet, M., Kiers, H. A., Van den Noortgate, W., \& Ceulemans, E. (2015). PCovR: An R Package for Principal Covariates Regression. Journal of Statistical Software, 65, 1-14. doi:10.18637/jss.v065.i08.

Vigil-Colet, A., Morales-Vives, F., Camps, E., Tous, J., \& Lorenzo-Seva, U. (2013). Development and validation of the Overall Personality Assessment Scale (OPERAS). Psicothema, 25, 100-106. doi:10.7334/psicothema2011.411.

Wolkowitz, A. A., \& Skorupski, W. P. (2013). A Method for Imputing Response Options for Missing Data on Multiple-Choice Assessments. Educational and Psychological Measurement, 73, 1036-1053. doi:10.1177/0013164413497016
Yuan, K. H., \& Lu, L. (2008). SEM with missing data and unknown population distributions using two-stage ML: Theory and its application. Multivariate Behavioral Research, 43, 621-652. doi:10.1080/00273170802490699.

Yuan, K. H., \& Savalei, V. (2014). Consistency, bias and efficiency of the normal-distribution-based MLE: The role of auxiliary variables. Journal of Multivariate Analysis, 124, 353-370. doi:10.1016/j.jmva.2013.11.006.

Yuan, K. H., \& Zhang, Z. (2012). Robust structural equation modeling with missing data and auxiliary variables. Psychometrika, 77, 803-826. doi:10.1007/s11336-012-9282-4

Yuan, K. H., Marshall, L. L., \& Bentler, P. M. (2002). A unified approach to exploratory factor analysis with missing data, nonnormal data, and in the presence of outliers. Psychometrika, 67, 95-121. doi:10.1007/BF02294711.

(Article received: 17-12-2014; revised: 23-02-2015; accepted: 03-03-2015) 